\title{
Does Muti-media Health Education Reduce the Workload of Nurses Without Affecting Patient's Satisfaction: A study protocol for a randomized controlled trial
}

\section{Liping Yang ( $\sim 114559573 @ q q . c o m)$}

Department of General Surgery, the First Hospital of Lanzhou University

\section{Huaping Wei}

Lanzhou University First Affiliated Hospital

Caiyun Zhang

Lanzhou University First Affiliated Hospital

\section{Zhigang Zhang}

Lanzhou University First Affiliated Hospital

\section{jINHUI Tian}

Lanzhou University

\section{Xiaojia Ma}

Chinese Academy of Sciences

Iulu Han

Lanzhou University

$\mathrm{Na} \mathrm{Li}$

Lanzhou university

\section{Wenbo Meng}

Lanzhou University First Affiliated Hospital

Jia Yao

Lanzhou University First Affiliated Hospital

\section{Study protocol}

Keywords: Muti-media Health Education, nurse workload, surgical patients, patient satisfaction

Posted Date: April 8th, 2021

DOI: https://doi.org/10.21203/rs.3.rs-68430/v1

License: (9) This work is licensed under a Creative Commons Attribution 4.0 International License. Read Full License 
Page 2/16 


\section{Abstract}

Background: Health education is an educational activity, which can help to improve the individuals and groups health care knowledge, establish basic health concepts and voluntarily adopt healthy behaviors and lifestyles through information dissemination and behavioral intervention. As one of the key influencing factors of patients' rehabilitation, health education with high quality and efficiency has been gaining more focus. Nowadays, health education in surgical intervention departments is mainly carried out by oral face-to-face communication and guidance from admission nurses; however, this is timeconsuming and laborious. In addition, oral guidance is influenced by the nurse's ability for expression and acknowledgement.

Methods: This is a protocol for a perspective, parallel-group RCT conducted at a Hepato-Pancreato-Biliary surgery institute in Lanzhou. A total of 184 patients aged $\geq 18$ years with general surgery illness and acceptable surgery, will be randomly allocated to 1 of the two groups (1:1 ratio). Conscious, cooperative Chinese speaking patients with primary school or above education level were recruited in the study. Each participant will have a baseline assessment at the hospital and the clinical data will be collected. In the group of multi-media video education, routine content of health education will be videotaped and played by the nurses on the first day of admission of the participants. Primary outcomes are nursing workload, and satisfaction of patients, family members, doctors and nurses. Secondary outcomes will include patients' anxiety level, inquiry time, time taken by the patients and their family members to watch videos.

Discussion: This study investigates the two types of health education methods for surgical patients. These results may help reduce the workload of nurses, without impacting the satisfaction of surgical patients.

Trial registration: NCT03989401. Registered on 17 June 2018.

\section{Introduction}

With the change of medical model, health education has become one of the most important contents of nursing work and runs through the whole work process. As the executor of health education, nurses need to master abundant medical knowledge and improve cooperation of patients for treatment and care.

A number of studies have demonstrated that surgery operations usually causes severe physical and mental stress on patients. Fear and anxiety due to the uncertainty of surgery may finally affect patient's surgical efficacy and recovery. Therefore, health education for perioperative patients has attracted great attention of many medical experts and clinical psychologists all around the world [1]. Many studies have shown that high quality health education can significantly reduce the perioperative fear, anxiety and pain of patients caused by surgical trauma and can reduce the incidence of various complications efficiently after surgery [2,3]. For this purpose, surgical nurses require more health education to reduce the mental and physical trauma after an operation. This is another reason that surgical nurses face much more workload than internal medicine nurses 
Health education constitutes the major part of nursing work in patient's medical management[4]. Health education methods satisfying patient's needs during perioperative period can not only arouse patient's interest in health education, but can also help the patients to correctly understand the relevant knowledge of disease and obtain training of the skills of recovery [5]. It plays an important role in patients' recovery of diseases and prevention of complications [6].

At present, health education in the department of surgery is mainly carried out through face-to-face oral communication and guidance from admission nurses, which is time-consuming and laborious [7]. In addition, oral guidance is influenced by the nurses' ability of expression and acknowledgement. Although a number of relatively good health education methods are available, such as reference books, communication apps such as WeChat [8-11], lack of comprehensive guidance and narrow application ranges make their clinical utilization limited. Information-based health education is a model combined with audio-visual stimulation and patient's own participation. Mobile terminals make patients gradually accept relevant health knowledge and functional exercise according to the hospitalization cycle, and at the same time, make health education work procedural, standardized and specific. Yang et al.[12] conducted a four-month study on comprehensive health education model for high-risk stroke patients (including watching videos, distributing health education manuals and lectures, simulating role-playing and personalized counseling), and concluded that this program had significant effects on improving patients' stroke medical knowledge increasing pre-stroke symptom warning and reducing the possibility of pre-hospital delay. Liu J et al.[13] also confirmed the use of WeChat public account in perioperative health guidance for parents of children undergoing herniorrhaphy, and showed that it improved the parents' awareness of perioperative period and help their children to prepare for surgery thus reducing the incidence of cancellations. Similarly, Amutha et al.[14] used intensive dietary intervention based on the Internet (12 courses updated every six months every two weeks) in type 2 diabetic patients, which showed that network intervention was a feasible way to implement chronic disease management. In another study [15] 25 minutes preoperative observation was applied to patients with coronary heart disease (including coronary atherosclerosis and coronary symptoms) and catheter insertion. Health education related to arterial surgery can familiarize patients with surgical complications, procedures and home care after discharge. This study showed that educational video intervention could effectively reduce the perioperative anxiety. However, there are only a few studies reporting the outcome of nurse's workload and patient's satisfaction with multi-media education. The aim of this study is to assess whether multimedia-video education can reduce the nurses' workload and without affecting the satisfaction of surgical patients.

\section{Aim}

The aim of the study is to determine the effect of multimedia health education in reducing the workload of nurses, without affecting the patients' satisfaction.

\section{Objectives and hypotheses}


. Determine if Multi-media health education may reduce the workload of nurses compared with conventional health education.

. Determine whether Multi-media health education does not decrease the satisfaction of patients, family members, doctors and nurses about health education in surgical ward on admission.

- Evaluate if Multi-media health education may reduce patient's' anxiety level on the second day of admission.

\section{Methods}

\section{Study design and sites}

This study is a perspective randomized controlled intervention trial with two arms (multimedia-video health education and usual health education) and four time points of data assessment (screening, baseline, the day of admission and the second day of admission), which will be conducted at a HepatoPancreato-Biliary surgery institute of Gansu province in China and will be conducted over approximately 12 months (between June 2019 and June 2020). A total of 184 participants with general surgery illness and who need surgery will be recruited.

Participants will be randomly allocated to receive either: (i) multimedia-video health education or (ii) conventional health education. The trial design is outlined in figure 1. Ethical approval for this trial has been obtained from the ethics committee of the First Hospital of Lanzhou University (registry number: LDYYLL2019-203). The trial is registered at Clinical Trials.gov (NCT03989401).

\section{Participants and selection criteria}

Participants with general diseases requiring surgical treatment are eligible for the study. The inclusion criteria for these index participants is as follows: (i) older than 18 years with general surgery diseases and need surgical treatment, (ii) with primary school or above education history, with clear awareness, (iii) can cooperate with the collection of clinical data, (iv) can communicate in Chinese, (v) signed the informed consent. Patients will be excluded if they have (i) visual and hearing impairment, (ii) mental illness, dementia or other mental disorders, (iii) complications of heart, brain and nephropathy, (iv) lack of selfcare ability (v) emergency and are critically ill, (vi) been previously included in other study.

\section{Assignment of interventions}

\section{Randomization and blinding}

Patients will be randomly assigned, using a random number table generated by SPSS software, to multimedia-video health education or convention health group with 1:1

allocation. The randomization numbers were independently stored and encoded in 184 sealed, opaque envelopes. When a patient meets the inclusion criteria, two nurses first record the name and the code of 
the next unopened envelope, and then open the envelope and sign their names

To ensure blinding of patients and study staffs, sealed randomization envelopes will be kept in a locked cabinet only accessible to study personnel who is not involved in measurements of outcomes. But nursing workload and the time of watching the health educational videos by the patients and their family members will be assessed by the researcher, responsible for this research.

\section{Interventions}

The First Hospital of Lanzhou University is a teaching hospital, where health education on admission is provided by nurses. All clinical nurses will be trained in relevant knowledge of health education before the start of the study.

\section{Multimedia-video health education}

According to the general surgical nursing routine, nurses will take the patients and/or family members into ward and explain how to use the multi-media video player to get the health education, then turn on the TV to play the videos on admission. The contents of health education video include: (i) hospital and ward environment, (ii) main clinical staff, (iii) safety precautions, (iv)hospitalization preparation, (v) visiting system, ward round and ask for leave system, (vi) routine medical examinations and precautions, (vii) dietary guidance and precautions, (viii) rehabilitation guidance and precautions, (viii) knowledge of taking medication and patient identification issues. Besides, they will tell the participants and their family members to consult the doctor or nurse in case of any queries. At the same time, experimental group and control group will live in separate rooms, to minimize mutual interference

\section{Usual care education}

Participants in the control group will get face-to-face oral health education by trained clinical nurses on admission. Contents of oral education are same as video, and nurses will also tell the participants that they can communicate with the doctor or nurse in case of any queries.

\section{Outcomes}

Primary outcomes are nursing workload and the satisfaction of patients, family members, doctors and nurses. These will be measured as following:

(1) To assess the nursing workload, working hours of nurses spent on the admission guidance in the first 24 hours of admission, and which include the time spent on guidance and addressing the patient's inquiry, collected by pre-designed forms.

(2) The satisfaction of patients and family members will be evaluated in the pre-designed questionnaire, which consists of 11 domains. These domains are: environment, disease, dietary, daily life and behavior, smoking situation and advice of giving up smoking, enhanced recovery after surgery, health examination, 
taking medicine, safety knowledge, duration of health education, and the kind of health education Every term of the questionnaire is represented by stars from one to five, also one to five scores, where one star represents unsatisfactory, and five stars represent highly satisfactory. The questionnaire is ranged from 11 to $55,11-21$ represents unsatisfactory, 22-32 represents not quite satisfactory, 33-43 represents generally satisfactory and 44-55 represents highly satisfactory.

(3) The satisfaction of doctor and nurse will be accessed by pre-designed questionnaires. \lt assesses doctor's and nurse's satisfaction after health education on admission under the following domains environment, disease, dietary, daily life and behavior, smoking situation and advise to give up smoking, rapid recovery, health examination, taking medicine, safety knowledge, duration of health education, and the way of health educationखservice attitude, professional knowledge, patient's condition and professional image. This questionnaire has 15 satisfaction questions, every term of the questionnaire is represented by stars from one to five, and also one to five scores, where one star represents unsatisfactory, and five stars represent highly satisfactory. The scores range from 15 to 75 , where a score of 11-29 represents unsatisfactory, a score of 30-44 represents not quite satisfactory, 45-59 represents generally satisfactory and a score of 60-75 represents highly satisfactory.

The secondary outcomes are patients' anxiety level before and after health education, patients' family members health education content enquire time, and the health education videos watching time.

(1) Patients' anxiety level before and after health education is measured by the Zung Self-Rating Anxiety Scale (SAS) [16], which is designed by William WK Zung to quantify the level of anxiety for patients experiencing anxiety related symptoms. The SAS consists of twenty questions which are scored as 1-4 points individually ( 1 , rarely; 2 , some of the time; 3 , very often/often; 4 , almost/always) and have fifteen questions about increasing anxiety levels and five questions about decreasing anxiety levels, resulting in 20-80 raw score. The total raw score consequently has to be converted to an "Anxiety Index". The clinical interpretation of one's level of anxiety is 20-44 represents normal range, $45-59$ represents mild to moderate anxiety levels, 60-74 represents marked to severe anxiety levels and 75-80 represents extreme anxiety levels.

(2) The time taken by patients or their family members, in inquiry and watching the health education videos, are recorded by a pre-designed table.

\section{Participant timeline}

Please see table 2.

\section{Sample size}

Sample size and statistical power has been calculated by health education Group AB of Statistical Analysis Software (SPSS), based on the previous studies ${ }^{[17]}$ assessing the satisfaction of health education. A power calculation was based on the primary outcome of satisfaction score on the health 
education of patients. Previous studies $[18,19]$ have demonstrated that a difference in the satisfaction score on the health education of patients between two groups constitutes a clinically meaningful change in satisfaction. Based on the computational formulae to compare the two means and other earlier findings regarding effects of satisfaction of health education, we estimated that a sample size of $\mathrm{N}=184$ (i.e. $n=92$ participants in each group) will reach $a \geq 80 \%$ power to detect a similar clinically relevant difference in the primary outcome measure between the treatment groups at an alpha level of 5\% (twosided test).

\section{Statistical analysis}

After the data are entered into Excel, the statistician will check the input data in detail to ensure the rationality and integrity of the data. After the inspection, data will be imported into SPSS 22.0 software for the statistical analysis. All statistical tests will be performed using a two-sided test and $p$-value less than 0.05 (two tailed) will be considered statistically significant. Quantitative data will be summarized using mean and standard deviation $(M \pm S D)$, qualitative data will be expressed as frequency distribution, and Kolmogorov-Smirnov test will be used to assess whether normal distribution is satisfied. Chi-square test will be used to analyze the relationship between independent qualitative data, $t$-test will be used to compare the mean values between the two groups for quantitative data satisfying normal distribution, and rank sum test will be used to compare the average values of the two groups for quantitative data not satisfying normal distribution. Analysis of variance will be implemented to compare the continuous variables between the groups.

\section{Recruitment}

The recruitment would last 12 months and began in August 2019. This duration was estimated based on the number of patients admitted to the Special Surgery Department of the first hospital of Lanzhou University, in the past 2 years. To obtain the adequate participant enrollment, relevant work on the study would be performed around the clock, including the nights and weekends.

\section{Data management and monitoring}

Specially trained study staff will collect the data required for the trial. The research staff will interview each participant at baseline to collect the data on patient characteristics and conduct the assessments for those questionnaire-based outcomes. During the interview, participant will receive paper-based assessment forms (questionnaires), and will be given adequate time for the completion of these. The completeness of each questionnaire will be checked at the end of the interview by the research staff for the quality purposes. To reduce study staff bias, study staff will be retained and supervised weekly to ensure the optimum degree of comparability. The source of information will be the patients and their family members. Data will be recorded on a case table at the time of assessment and data collection will be held at the hospital. All data are controlled by two people and entered into the computer. This allows for plausibility checks at the time of input and will avoid the errors that would otherwise result from transferring the data from written notes to a computer. Written informed consent forms will be kept in a 
locked filing cabinet, while a password-protected list that matches participant ID numbers with personal information will be stored separately from pseudo-anonymized data. List matching participants' personal information with their ID number will be deleted and consent forms maculated 5 years after study completion. At this point, all data will be completely anonymous. All trial authors will have access to the ultimate trial dataset.

\section{Harm, and auditing}

Serious and nonserious adverse events, considered related to the trial, will be rigorously and carefully recorded. A auditing committee consisting of a surgeon and a nurse will supervise the trial at least once every month. They remain independent audit the frequency, procedures, blind and safety of the trial.

\section{Quality assurance and safety}

Research and Design Phase

(1) At the stage of research and design, communicate with qualified teachers, discuss the feasibility of research methods and ensure the correct implementation of each link. (2) Before the start of the study, all researchers should be able to understand and master the purpose, content, steps and precautions of the study, and should be tested. After the start of the study, follow-up guidance should be provided to the researchers to ensure the quality of the study.

Data collection stage

(1) Formulate the strict inclusion and exclusion criteria, and to select research objects strictly according to the inclusion criteria. (2) During the course of the study, the researchers give accurate records of the health education and the patient's condition every day. (3) All the members of this research group are nurses that have worked in the department of general surgery for more than five years and have received special health education training. The members of the research group include instructors, researchers, data collectors and statisticians. They have a clear division of the labor and all work together. (4) Subject group members regularly give feedback on the research, discuss the existing problems and make plans for the next month.

Data entry and analysis stage

(1) On the data input stage, all data will be checked by two people and entered, and statistical analysis will be carried out on the premise of ensuring the correctness of data.

(2) In the process of data analysis, all are instructed to listen to the advice of the professional statistical instructor.

\section{Ethical considerations}


This is a clinical experimental study and will not cause substantial moral hazard. In the process of research, the following principles would be strictly observed: (1) the principle of informed consent would be followed. Only those subjects will be included who understand the nature and purpose of the study and voluntarily agree to participate and sign a written informed consent. (2) Patients would have the right to refuse participation and withdrawal at any time. (3) This research would be beneficial for the patients' disease recovery and physical and mental development. (4) The researchers would keep the personal information obtained in this research confidential. When research results are published publicly, no matter whether the patients agree or not, are all aliases, and the real name will not appear.

\section{Protocol amendments}

Any changes to the inclusion criteria, outcomes, and analyses would require approval from the ethics committee and the clinical trial.

\section{Confidentiality}

Data would be treated in a confidential manner. During the whole experiment, the patient's name, hospitalization number, and other sensitive information would not be exposed through coding.

\section{Access to data}

All investigators will have access to the final trial data set after the end of the study.

\section{Dissemination}

Results will be presented at academic conferences and published in peer-reviewed journals. We will inform the participants of the results via E-mail.

\section{Discussion}

With the change of medical model, health education has become one of the important components of the nursing work. As the executor of health education, nurses must master the medical and health education knowledge so as to enable patients to cooperate actively with treatment and nursing. To know whether multimedia health education can meet the needs of high quality, high efficiency and low-cost nursing is the main purpose of this study.

A large number of studies at home and abroad have shown that most surgical patients have different degrees of psychological stress reaction against perioperative periods. Therefore, health education in such patients has attracted great attention for many medical experts and clinical psychologists at home and abroad [20]. Studies have shown that good health education can significantly reduce the perioperative patients' fear, anxiety and pain caused by surgical trauma, and achieve disease prevention and treatment and reduce the incidence of various complications after surgery. Providing health education to the patients is also an important part of the medical management. Providing health 
education, using a method oriented to patients' needs during perioperative periods, can not only arouse patients' interest in the health education, but also improves patients' awareness about the diseases. It is of great significance to promote the recovery of the patient and in the prevention of complications

However, the traditional way of health education is one-to-one oral explanation, which is boring and lacks uniform norms and standards. Moreover, due to the differences in professional level, language expressive ability and emotional communication ability of nurses, there may be big deviations in the content and effect of different levels of nurses' education [21]. In addition, due to the difference in patients' age, gender, education, emotional and physical conditions, the acceptance of health education content is different. Although there are some image health education methods, such as booklet [22,23], WeChat[24,25], the lack of comprehensive guidance, and narrow application scope, makes them non conducive to clinical popularization. Multimedia video is as an effective health education method which has been widely used in other medical and health fields, such as for cardiovascular disease (CVD) patient's [26] health education. Studies have shown that [27] video presentation is easier to understand than the simple oral presentation. Patients gain knowledge from the healthy education video which enables them to get a more accurate cognition of the surgical process, to develop disease selfmanagement ability ${ }^{[10]}$, and reduce perioperative anxiety.

Health education, using advanced technology such as multi-media to manage an individual or a group, improves the quality and effect of the clinical health education. It realizes the personalized needs of the health education object, and makes a systematic closed-loop mode to achieve the maximum health benefits using limited resources, so as to help medical staff better evaluate and manage the surgical patients. The aim of this study was to compare the effectiveness of the multimedia-video health education with conventional methods to educate the patients in surgical wards upon admission, in order to find out higher quality, efficient and low-cost health education program for the surgical patients, and then improve the connotation of nursing, so that the effect and efficiency of high-quality nursing services can be improved most. We hypothesize that information health education could reduce nurses' workload while not affecting the patients' satisfaction. The main limitation of this study is as follows: (1) A multicenter study was not conducted and the informationized health education for internal medicine patients was not adopted; (2) no specific health education methods was implemented for the different diseases and populations.

\section{Strengths And Limitations Of This Study}

- Integration of a multi-media system with a clinical health education care service can potentially improve the nurses' workload.

- The multi-media health education system delivers core recommendations for patients in the surgical ward, evident by the enhanced recovery after surgery (ERAS). 
- Specific health outcomes of relevance to the multi-media health education system will be measured in this study.

- The study is limited to a single-center study, and as a purely surgical patients, the multi-media health education does not include different diseases and populations.

\section{Trial Status}

The LCEVP protocol version is 3.0 (as of 15 May 2019). The trial started recruitment on 10 August 2019. Maybe we would complete the recruitment on 10 August 2020.

\section{Abbreviations}

LDYYLL: Ehics committee of the first hospital of Lanzhou University, NCT: Clinical Trials. Gov, ERAS: Enhanced recovery after surgery, SAS: Anxiety Scale, SPSS: Statistical Analysis Software, M \pm SD: Mean and standard deviation.

\section{Declarations}

\section{Acknowledgements}

The authors would like to thank all those who helped them write the thesis, all professors in the nursing department of the first hospital of Lanzhou University, and Chunfei Huang for their assistance during the shooting and editing of the health education video.

\section{Authors' contributions}

LPY participated in the protocol development, drafting of this manuscript, and critical revision of the manuscript regarding the intellectual content. JY, WBM, and CYZ participated in the design of the protocol and the methodological development. WBM and JY were responsible for the sample size calculation and statistical

analysis. HPW, ZGZ, and JHT participated in the design of the study, the methodological development, and the logical interpretation and presentation of the results. LLH, XJM, and NL participated in the study's design and reviewing the article for spelling and grammar as well as its intellectual content.

\section{Funding}

The main funding sponsor is the Hepatopancreatobiliary Surgery Institute of Gansu Province. This study was financially supported by the health industry research program in the Gansu Province (Grant No. GSWSKY-2019-46). The authors and investigators did not receive any funding from the commercial partners. The study's sponsor and funders did not have any involvement in the study design, analysis, 
management, collection and interpretation of the data, writing the manuscript, or the decision to submit the report for publication.

\section{Availability of data and materials}

Results are expected to be published in a medical journal and will be available for enrolled patients who wish to learn about the outcome.

\section{Ethics approval and consent to participate}

The study was registered at http://www.clinicaltrial.gov on 3 July 2019 with trial identification number NCT03989401. Ethics Committee of The First Hospital of Lanzhou University approved the study in 2019 and the approval Number is LDYYMENG: 2019-203.

All participants will require to provide written informed consent. We shall seek written informed consent from next of kin if the patient cannot provide one due to the severity of the illness and only continue the trial when the patient agrees to, in writing, after his/her condition is restored.

\section{Consent for publication}

Not applicable.

\section{Competing interests}

The authors declare that they have no competing interests.

\section{References}

1. Menendez ME, Ring D. Does the timing of surgery for proximal humeral fracture affect inpatient outcomes? J Shoulder Elbow Surg 2014; 23(9):1257-62.

2. Pritchard MJ. Managing anxiety in the elective surgical patient. British Journal of Nursing (Mark Allen Publishing) 2009; 18(7):416-9.

3. Chang SF, Hung CH, Hsu YY, et al. The Effectiveness of Health Education on Maternal Anxiety, Circumcision Knowledge, and Nursing Hours: A Quasi-Experimental Study. Journal of Nursing Research 2017; 25(4):1.

4. Hassan NM, Win KT, Hyland P. Exploring design features and benefits of Online Patient Education (OPE) sites for chronic diseases. In: System Sciences (HICSS), 2013 46th Hawaii International Conference on, 7-10 Jan. 2013; pp 2454-2463. doi:10.1109/HICSS.2013.215, 2013

5. Hounsome J, Lee A, Greenhalgh J, et al. A systematic review of information format and timing before scheduled adult surgery for peri-operative anxiety. Anaesthesia 2017; 72(10):1265-1272.

6. Win KT. Implementing patient accessible health information site for diabetes management. Paper presented at the Pacific Asia Conference on Information Systems. National Taiwan University: 
Taiwan, 2010.

7. Wang J, Tian J. Evaluation of the Effect of Nursing Quality Indicator Management in Nursing of Critical Patients. General Journal of Stomatology 2018, v.5(14):76-77.

8. Zhang X, Xi W, Liu L, et al. Improvement in Quality of Life and Activities of Daily Living in Patients with Liver Cirrhosis with the Use of Health Education and Patient Health Empowerment. Medical Science Monitor 2019,25, 4602-4608. doi:10.12659/MSM.914487

9. Kakinuma A, Nagatani H, Otake H, et al. The Effects of Short Interactive Animation Video Information on Preanesthetic Anxiety, Knowledge, and Interview Time: A Randomized Controlled Trial[J]. Anesthesia \& Analgesia 2011, 112(6):1314-8.

10. Lattuca B, Barber-Chamoux N, Alos B, et al. Impact of video on the understanding and satisfaction of patients receiving informed consent before elective inpatient coronary angiography: A randomized trial[J]. American Heart Journal 2018: S0002870318300784.

11. Helen CT, Richard WB, Lyn J, et al. A randomized controlled trial of telephone-mentoring with homebased walking preceding rehabilitation in COPD. Int J Chron Obstruct Pulmon Dis 2016, 11:19912000.

12. Yang L, Zhao Q, Zhu X, et al. Effect of a comprehensive health education program on pre-hospital delay intentions in high-risk stroke population and caregivers. HYPERLINK "https://www.ncbi.nlm.nih.gov/pubmed/28401417" Io "Quality of life research : an international journal of quality of life aspects of treatment, care and rehabilitation." Qual Life Res2017;26(8):21532160.

13. Liu J, Zheng X, Chai S, et al. Effects of using WeChat-assisted perioperative care instructions for parents of pediatric patients undergoing day surgery for herniorrhaphy. HYPERLINK "https://www.ncbi.nlm.nih.gov/pubmed/29499997" Io "Patient education and counseling." Patient Educ Couns2018;101(8):1433-1438.

14. Ramadas A, Chan CKY, Oldenburg B, et al. Randomized-controlled trial of a web-based dietary intervention for patients with type 2 diabetes: changes in health cognitions and glycemic control. BMC Public Health 2018, 18(1):716.

15. Ayasrah SM, Ahmad MM. Educational Video Intervention Effects on Periprocedural Anxiety Levels Among Cardiac Catheterization Patients: A Randomized Clinical Trial. Res Theory Nurs Pract 2016, 30(1):70-84.

16. Knight RG, Waal-Manning HJ, Spears GF. Some norms and reliability data for the State-Trait Anxiety Inventory and the Zung Self-Rating Depression scale. Br J Clin Psychol1983;22 (Pt 4):245-9.

17. Atack L, Luke R. The Impact of Validated, Online Health Education Resources on Patient and Community Members' Satisfaction and Health Behaviour. Health Education Journal 2012, 71(2):2118.

18. Blaasvaer S, Laerum E. [Effects of a health-education approach on health behavior, consultation satisfaction and psychological wellbeing]. Tidsskrift for Den Norske Lægeforening Tidsskrift for Praktisk Medicin Ny Række, 1998, 118(3):370. 
19. Kim JH, Yoo KB, Park EC, et al. Combined effects of education level and perceived social class on self-rated health and life satisfaction: Results of Korean labor and income panel study wave 8-wave 15. Health Qual Life Outcomes 2015, 13(1):178.

20. Bruckenthal P, Simpson MH. The Role of the Perioperative Nurse in Improving Surgical Patients' Clinical Outcomes and Satisfaction: Beyond Medication. AORN J 2016, 104(6S): S17-S22.

21. Chen Huajuan, Chen Limin, Chen Caiping,Application of Video Interactive Education Model in Health Education for Puerpera's Spouses.nursing journal of Chinese police's liberation army, 2018, v.35区 376(10):80-82.

22. Yu M, Chair SY, Chan CWH, et al. A health education booklet and telephone follow-ups can improve medication adherence, health-related quality of life, and psychological status of patients with heart failure. Heart Lung 2015, 44(5):400-7.

23. Vardanjani SAE, Fanisaberi L, Shahraki The Effect of Face-to-Face Education and Educational Booklet on Heart Health Indexes of the Hospitalized Patients with Myocardial Infarction. Nursing Research and Practice 2013, 2013:675634.

24. Dong $Y$, Wang $P, D a i Z$, et al. Increased self-care activities and glycemic control rate in relation to health education via WeChat among diabetes patients: A randomized clinical trial[J]. Medicine, 2018, 97(50).

25. Liu J, Zheng X, Chai S, et al. Effects of using WeChat-assisted perioperative care instructions for parents of pediatric patients undergoing day surgery for herniorrhaphy[J]. Patient Education \& Counseling, 2018:S0738399118300752.

26. Gagné, Myriam, Legault $C$, Boulet $L P$, et al. Impact of adding a video to patient education on quality of life among adults with atrial fibrillation: a randomized controlled trial[J]. Patient Education and Counseling, 2019;102(8):1490-1498.

27. Harkness, K. The effect of early education on patient anxiety while waiting for elective cardiac catheterization[J]. European Journal of Cardiovascular Nursing, 2003, 2(2):113-121.

\section{Table}

Table 1 is not available in this version.

Due to technical limitations, Table 2 is only available as a download in the supplementary files section.

\section{Figures}




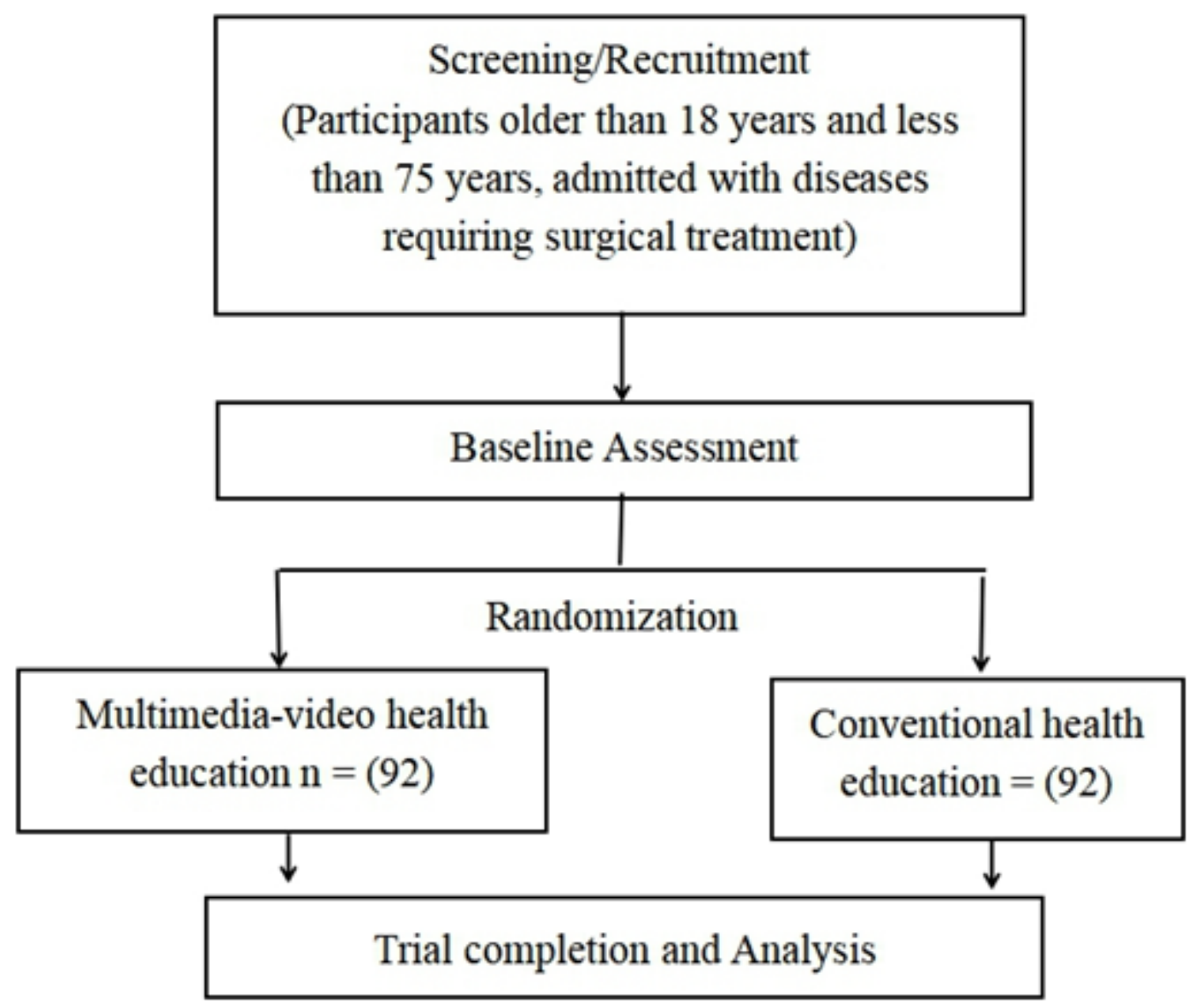

Figure 1

Flow diagram for the health education trial

\section{Supplementary Files}

This is a list of supplementary files associated with this preprint. Click to download.

- SPIRIT2013Checklist.doc

- Table2.pdf 